\title{
BMJ Open Living strategies for disability in men ageing with HIV in Ontario, Canada: a longitudinal qualitative study
}

\author{
Patricia Solomon, ${ }^{\circledR 1}$ Kelly K O'Brien, ${ }^{\oplus 2}$ Rebecca McGuff, ${ }^{1}$ Michelle Sankey ${ }^{1}$
}

To cite: Solomon P, O'Brien KK, McGuff R, et al. Living strategies for disability in men ageing with HIV in Ontario, Canada: a longitudinal qualitative study. BMJ Open 2019;9:e031262. doi:10.1136/ bmjopen-2019-031262

- Prepublication history for this paper is available online. To view these files, please visit the journal online (http://dx.doi. org/10.1136/bmjopen-2019031262).

Received 24 April 2019 Revised 01 August 2019 Accepted 12 August 2019
A) Check for updates

(C) Author(s) (or their employer(s)) 2019. Re-use permitted under CC BY-NC. No commercial re-use. See rights and permissions. Published by BMJ.

${ }^{1}$ School of Rehabilitation Science, McMaster University, Faculty of Health Sciences, Hamilton, Ontario, Canada ${ }^{2}$ Department of Physical Therapy, University of Toronto, Faculty of Medicine, Toronto, Ontario, Canada

Correspondence to Dr Patricia Solomon; solomon@mcmaster.ca

\section{ABSTRACT}

Objectives To examine the living strategies used by older men living with HIV to deal with the episodic nature of disability and associated uncertainty, over time.

Design Qualitative longitudinal study in which men living with HIV were interviewed on four occasions over 20 months.

Setting Men were recruited from HIV community organisations in Canada.

Participants 14 men with a median age of 57.5 years and median time since diagnosis of 21.5 years.

Results Five themes depict the living strategies used to deal with the episodic nature of disability and uncertainty over time. Actively engaging in problem-solving by prioritising and modifying activities and avoiding stress, advocating for support, being positive and future oriented, engaging in healthy pursuits and providing social support to others helped men to mitigate their disability. By following participants over time, we were able to gather insights into triggers of episodes of disability, and perceptions of the success of implementing their living strategies and avoiding uncertainty.

Conclusions Participants used living strategies to deal with uncertainty and mitigate episodes of disability over time. This study supports the importance of programmes that promote self-management for older men living with HIV through helping them identify triggers of disability, set realistic goals and problem-solve. These may help build self-efficacy, increase sense of control, and decrease feelings of uncertainty and episodes of disability.

\section{INTRODUCTION}

In resource rich countries where people living with HIV are growing older and attaining a near normal life span, there has been increased recognition of HIV as a chronic illness that may result in health challenges or disability. ${ }^{1-4}$ In Canada, the prevalence of older adults ( $\geq 50$ years) living with HIV is increasing due to ageing of long-term survivors and an increase in new HIV diagnoses among this older age group. ${ }^{5}$ Older adults living with HIV may experience disability related to the virus, side effects of medication, multimorbidity and natural consequences of ageing. ${ }^{6}$ This disability may be compounded

\section{Strengths and limitations of this study}

We conducted a longitudinal qualitative study of the living strategies used by older men living with HIV over time.

- We interviewed participants on four occasions over a 20 -month time frame and achieved a $100 \%$ retention rate.

- Strengths included prolonged engagement of participants over time and use of a disability lens as a foundation for this research.

- Limitations include an independent community-dwelling sample of long-term survivors which may be better able to access supports and engage in positive living strategies.

by significant social problems, stigma, mental health issues and social isolation. ${ }^{78}$ Given this is the first cohort to grow old with HIV, it is important to understand the complexity of their disability.

The disability experienced by people living with HIV is often episodic in nature, associated with unpredictable fluctuating periods of good and ill health. ${ }^{9}$ The Episodic Disability Framework, derived from people living with HIV, includes four dimensions of disability: symptoms and impairments, difficulties carrying out day to day activities, challenges to social inclusion and uncertainty that can be influenced by extrinsic and intrinsic contextual factors. ${ }^{9}$

While a key dimension in the Episodic Disability Framework, uncertainty resulting from unpredictable fluctuations in health is also viewed as a defining feature of ageing with HIV that can increase anxiety of their everyday life. ${ }^{10}$ Uncertainty is at the centre of a model of disability influencing symptoms, activity and participation levels for older adults living with HIV. ${ }^{11}$ Further modelling of dimensions of disability has suggested uncertainty is a direct and strong predictor of mental and emotional health challenges and challenges to social inclusion for people living with HIV. ${ }^{12}$ Age-related uncertainties 
include worries about the source of health challenges, concerns about health providers' knowledge of HIV, financial and retirement concerns and long-term care and housing options. ${ }^{13}$ Furlotte and Schwartz ${ }^{14}$ reinforced that uncertainty contributes to mental distress among older adults living with HIV. Kylmä et $a l^{15}$ hypothesise that uncertainty can be both negative and positive; if combined with fear it may lead to hopelessness, while combined with wishing, is related to hope.

While a number of studies have reviewed how people living with HIV cope with a chronic illness,${ }^{16}$ few have addressed how they deal with the episodic nature of disability and associated uncertainty. Brashers et $a l^{17}$ found that social support helped people living with HIV to manage uncertainty through a variety of strategies such as assisting with information-seeking, providing instrumental support and offering acceptance. Uncertainties related to HIV are perceived differently by health professionals and people living with HIV, with professionals viewing uncertainty as related to a lack of scientific knowledge, and people living with HIV and policy-makers viewing uncertainties as chronic stressors. ${ }^{10}$

In the Episodic Disability Framework, living strategies are intrinsic contextual factors that may interact and influence dimensions of disability including uncertainty. ${ }^{9}$ The term living strategies was derived from the perspective of people living with HIV $^{9}$ and is analogous to coping in the context of HIV. ${ }^{18}$ While there is some conceptual overlap, living strategies are differentiated from coping in that these can have both positive and negative influences on health. ${ }^{18}$ A greater proportion of older adults with HIV engage in living strategies focused on maintaining a sense of control over one's health and adopt positive attitudes compared with younger adults with HIV. ${ }^{18}$

Studies examining coping and living strategies in older adults living with HIV have predominantly been cross-sectional in nature. Few have examined how the episodic nature of the disability, including the dimension of uncertainty, may be influenced by living strategies used by people living with HIV. Episodic disability is a temporal phenomenon best understood through longitudinal inquiry. Our goal was to answer the following research question: what is the nature and extent of living strategies used by older adults living with HIV to manage the episodic nature of disability, and associated uncertainty, over time?

\section{METHODS}

We conducted a longitudinal qualitative study involving a series of four, semi-structured face-to-face interviews with older men and women living with HIV. This was a part of a larger study of 24 participants (14 who identified as men and 10 who identified as women). Each participant was engaged over a 20-month time frame.
Interviews were scheduled 5 months apart to allow for changes in function and disability to occur but short enough to maintain momentum and promote retention. 1920

Participants were recruited through HIV community organisations in Southern Ontario, through pamphlets onsite and recruitment notices on websites. Eligible participants were 50 years of age or older, who were diagnosed with HIV for more than 6 years. We excluded those newly diagnosed ( $<6$ years ago) as this population may not have experienced ill health and/or disability associated with long-term survival. ${ }^{921}$ There are gender differences in the strategies people living with HIV use to deal with their health challenges. ${ }^{22-24}$ Thus, in this study we present the analysis of the participants who identified as men.

We used the Episodic Disability Framework ${ }^{9}$ to guide the interviews. During the first interview (time 1), we asked participants to provide a general description of their health challenges (or disability) including physical, cognitive, mental and emotional symptoms and impairments, difficulties carrying out day to day activities, uncertainty and challenges to social inclusion. The challenges were then explored in detail, probing for whether these were episodic in nature and the living strategies used to address their health challenges. In subsequent interviews, we explored the dimensions of disability identified in time 1 and asked participants what changes occurred, how these occurred and how these changes affected their functioning and health. Our design allowed for emergent themes to be discussed over time. Participants received an honorarium at the completion of each interview. We collected demographic data at time 1 and asked participants to complete the 20-item HIV Symptom Index which describes the number of symptoms present, and bothersome, to the individual. ${ }^{25}$

Analysis: All investigators contributed to the analyses. Interviews were audio-recorded, transcribed verbatim and entered into QDA Miner V.5 for data management. ${ }^{26}$ Longitudinal qualitative analysis requires summary and comparison of data both longitudinally and cross-sectionally. ${ }^{27}$ Initially, two investigators (RM and MS) independently carried out line by line coding of all four transcripts from one participant and developed a code book to guide our analysis. ${ }^{28}$ The analysis focused on identifying disabilities, the living strategies used to deal with their challenges, triggers of episodes and whether the disability was episodic in nature (varied over the interviews). Codebook development was an iterative process involving ongoing discussion and revision. All transcripts from three participants were then reviewed independently with a subsequent meeting with a third investigator (PS) to refine the codebook and resolve discrepancies. The remaining transcripts were then each independently coded by two investigators (RM and MS) using the codebook. Verification of coding and themes was an iterative process discussed by all investigators at team meetings. ${ }^{29}$ As we interviewed each participant on four occasions, we were able to follow the strategies used to address health challenges (or disability) and uncertainty 
over time. We developed an in-depth summary of each participant's disability experiences over time and compared these across participants to identify themes. All investigators reviewed and came to consensus on the final themes.

\section{Patient and public involvement}

This study is a part of a larger study in which a community member was included on the research team as one of the investigators. He reviewed and provided input on the research question and design prior to grant submission. Results of this study will be distributed to participants who indicated that they would like a copy of the research findings and provided contact information.

\section{RESULTS}

Fourteen men volunteered to participate in this study and completed all four interviews. Participant age ranged from 50 to 73 years (median: 57.5 years; IQR 53.3-65.3) with a median time since diagnosis of 21.5 years (IQR 19.8-25). Demographic data are presented in table 1. We identified five themes which illustrated the living strategies used by participants to manage uncertainty and minimise disability over time.

\section{Prioritising activities, modifying expectations, avoiding stress} Participants described problem-solving and developing strategies to manage their disability. They experimented with many strategies over time and were able to evaluate the most successful options. Participants described cognitive strategies to avoid dwelling on uncertainty and worrying about the future, as stated by this participant:

...rather than worry about what might, what could (be)... (I am) just dealing with the thing when it comes up. I seem to be much better at not worrying about it or not getting depressed over it. (Participant

5, age 66; living with HIV (LWH) 20 years)

Participants described how they prioritised needs and activities, and set goals. For example, many were on a limited income and tried to proactively budget so that they were able to meet their nutritional and social needs. Participants stated how acceptance of their limitations helped to prioritise activities. For example, modification of tasks was a way to live with fatigue. This participant recognised his limitations and described how he felt it was possible to do activities he did when younger:

(if you) change your strategy of how you accomplish it. (Participant 6, age 65; LWH 20 years)

Participants spoke of needing a 'different approach' and adjusting their expectations of what they could accomplish. One participant described how he had stopped volunteering at a hospital because he found it exhausting, stating:

at this point I need to give more to myself than to others. (Participant 11, age 51; LWH 25 years).

\begin{tabular}{|c|c|}
\hline Variable & $\begin{array}{l}\text { n (\%) or median } \\
\text { (IQR) }\end{array}$ \\
\hline Age (years) & $57.5(53.3-65.3)$ \\
\hline $\begin{array}{l}\text { Median time since HIV diagnosis } \\
\text { (years) }\end{array}$ & $21.5(19.8-25)$ \\
\hline \multicolumn{2}{|l|}{ Sexual orientation } \\
\hline Gay & $12(85.7)$ \\
\hline Bisexual & $1(7.1)$ \\
\hline Straight & $1(7.1)$ \\
\hline \multicolumn{2}{|l|}{ Current marital/partnership status } \\
\hline Single & $9(64.3)$ \\
\hline Married/living together & $4(28.6)$ \\
\hline Separated/divorced & $1(7.1)$ \\
\hline \multicolumn{2}{|l|}{ Level of education } \\
\hline High school or less & $4(28.6)$ \\
\hline College diploma/university degree & $7(50)$ \\
\hline Postgraduate education & $2(14.3)$ \\
\hline Other & $1(7.1)$ \\
\hline \multicolumn{2}{|l|}{ Estimated annual family income (\$C) } \\
\hline$<30000$ & $10(71.4)$ \\
\hline $40000-70000$ & $2(14.3)$ \\
\hline$>100000$ & $1(7.1)$ \\
\hline Missing & $1(7.1)$ \\
\hline \multicolumn{2}{|c|}{ Employment status (multiple responses permitted) } \\
\hline Working full-time or part-time & $5(35.7)$ \\
\hline Unemployed & $3(21.4)$ \\
\hline Volunteer & $4(28.6)$ \\
\hline Retired & $4(28.6)$ \\
\hline Student & $1(7.1)$ \\
\hline \multicolumn{2}{|l|}{ HIV Symptom Index } \\
\hline Total symptoms & 10.5/20 (8-15) \\
\hline Bothersome symptoms & $10.5 / 20(5.8-12)$ \\
\hline
\end{tabular}

Another participant described how letting "stuff" pile up could trigger depression and how he learnt to decide whether he should engage in an activity:

I'm getting much better at doing (a cost-benefit analysis) and saying, okay, it's of more benefit for me to do it, even though it's going to be a little bit uncomfortable, than it's going to be not to do it... (Participant 5 , age 66 ; LWH 20 years)

Often, a key to solving problems was the ability for participants to identify triggers of episodes of disability or ill health. Participants recognised stressors in their lives related to their illness, such as life and family situations which were triggers for mental health issues and could lead to activity limitations. Proactive stress avoidance was common. For example, participants described how they 
avoided individuals who did not contribute positively to their emotional well-being.

Through increased awareness of triggers of episodes of disability, many were able to prioritise activities, avoid uncertainty and mitigate an exacerbation. Weather was described as a trigger of depression and knowing this enabled participants to 'deal with it'. Fatigue was a common experience and participants described how they needed to be 'careful' to ensure that they had adequate energy levels. This participant described a 3-day regimen to ensure he was rested and avoided depression.

(you can) roll around and relax and eat chocolates and feel sorry for yourself...but then after the third day you've got to get out of your environment whether it be your apartment or your head space and try to just go for a walk or do a bit of exercise. (Participant 3 , age 61 ; LWH 30 years)

\section{Self-advocating for resources, care and support}

The ability to advocate for resources, both medical and financial, was noted as a key strategy to manage challenges from disability. Participants described how remaining active and involved with their health provided a sense of control and stability which circumvented feelings of uncertainty. Participants reflected on how important it was to manage their health, with many taking an active role in learning about their medications and side effects, management strategies and co-morbidities. They negotiated around how often they would need to access the health system and their preferences for health providers. This participant described the role he preferred his family doctor to play in management of his overall health:

I'll just talk to him next time I see him and say, 'You're my family doctor. I want you as my family doctor, you help me with a number of problems. But I don't want you to be my HIV doctor. Period. I am the centre of my care plan, not you.' (Participant 1, age 73; LWH 21 years)

Participants sought out information to learn about their health from the internet, community HIV organisations and health providers to play a more active role in their management. Negotiating care around multiple providers was often challenging. One participant described how after a prostate cancer diagnosis he wanted to meet with the specialist and his family physician separately so that he could make the most informed decision about treatment options. Participants recognised that it was important to be organised for healthcare visits, which included coming prepared with questions as this participant stated:

(I) started questioning the doctors. What's the medication going to do for me? What are the side effects? (Participant 10, age 65; LWH 20 years)
Participants stated knowledge about their medications related to HIV management, pain management and other co-morbidities including diabetes. They advocated with their physicians about how to decrease their pill burden. One participant spoke at time 3 of wanting to be off all psychiatric medications and reported he had done so at time 4.

Participants also described how they advocated for supports and resources to improve health and quality of life for themselves and for their communities. Several lived in subsidised housing and described working with tenant associations to deal with adverse situations or negotiating for changes to improve housing. For example, this participant described how he advocated for smoke alarms in his apartment building:

I looked up the law and it says very clearly that the manager or whatever is responsible for these things and I checked it out with the city. And I went down to our boss and said I would be pleased to see us come to a compliance with the Ontario fire code. (Participant 1, age 73; LWH 21 years)

\section{Looking to the future with positive outlook}

Participants described how their acceptance of ageing with their illness allowed them to be positive and future-oriented. Although older, many contemplated returning to school or retraining for a new job. Participants planned on travelling, fixing up their apartments, starting to exercise and diet, and becoming involved in volunteering. Participants were pleased to be growing older and thought beyond their limitations and past disappointments. One participant was excited about a new romantic relationship as he thought this would not happen at his age; as he stated:

...I was shocked that he was calling to ask me to go out for dinner. I thought, you must be joking. This stuff doesn't happen to me. And I'm talking over a quarter of a century (since I've dated). So it's a long, long time. (Participant 6, age 56; LWH 20 years)

The longitudinal design of the study allowed us to observe some participants' plans and goals come to reality. One participant dreamed of going to New York to see the ballet and the opera and at the second interview stated he had achieved his goal. Another participant's goal of volunteering emerged as an opportunity to be on panel discussions and developing videos for health science students. One participant set a goal of getting a bicycle and was able to acquire one between the second and third interviews. Another planned to increase his walking and persisted 'rain or shine'.

Participants associated their positive outlook with acceptance of growing older as noted by this participant:

( I am) not thinking of ageing, being old as an illness. It's simply a part of living. (Participant 1 , age 73 ; LWH 21 years) 
As long-term survivors with HIV, many participants reflected on their past challenges and contrasted this with their current status:

I think it's wonderful the medications that we have now, so that they can maintain a fairly substantial level of good health, for the long-term survivors. (Participant 6, age 65; LWH 20 years)

\section{Engaging in a healthy lifestyle}

Twelve men described healthy lifestyle choices and expressed their desire to continue to live a healthy life as they aged with HIV. Many were committed to healthy pursuits as a way of coping with ageing, HIV and comorbidity-related disability. Exercise and remaining physically active was the most common strategy articulated for maintaining health. With limited income, some found that walking was the most affordable exercise regimen. As this participant stated:

I walk as much as I can. Public transit when I can but as little as possible (Participant 5, age 66; LWH 20 years)

Many recognised when they needed external motivators to exercise regularly. Some joined the Young Men's Christian Association (YMCA) or a local gym. One participant started an outdoor walking programme for older adults with cardiac problems. Another received a bicycle which 'changed his life'. One joined a fitness study which included the services of a fitness coach which he found very motivating. Several attributed improvements in their mood and musculoskeletal pain to regular exercise.

Diet and weight concerns were common. Participants linked exercise and diet as a weight loss strategy. Many had goals of losing weight and some succeeded over time. Participants sought dietitian advice for co-morbidities such as diabetes and hypercholesterolaemia.

Other strategies for promoting a healthy lifestyle included learning to balance and pace activities to avoid fatigue. With increased activity several participants experienced more restorative sleep as noted by this participant:

(After going to the YMCA) I'm actually tired of doing something and I had a good sleep. And I actually sleep well. But when I didn't do the Y I wasn't sleeping properly. (Participant 4, age 54; LWH 25 years)

\section{Valuing reciprocity of social support}

Participants valued social support to help them deal with dimensions of disability (including uncertainty) and often sought to extend their circle of support. Support was received from many sources including family and friends, HIV community organisations, work and volunteer relationships, health and social services, and virtual networks. HIV community organisations were described as the 'main source' for support and as ways to access free activities (yoga class) or health and social services (nutritionist). Describing how he often felt depressed in the winter this participant stated:

But then as soon as I go to (the HIV community organization), everything changes. (Participant 7 , age 55; LWH 24 years)

Family members provided key supports, though several participants described dysfunctional family relationships over the duration of the study. Two participants stated that their support was 'sufficient' and that they preferred being alone with one stating:

I like to be by myself. (Participant 13, age 50; LWH 15 years)

The desire to also provide social support to others was an important aspect in the lives of many of the men. One participant described a complex relationship he had with an addicted homeless youth and how he tried to care for him. Another described how his family felt he was focusing too much on others and neglecting his own needs. When asked whether it was difficult to support others this participant remarked:

it takes your attention away from your own shtick. (Participant 2, age 67; LWH 24 years)

As participants were older, some dealt with the complexities of providing support to ageing parents. HIV community organisations provided a safe outlet for interaction and participants often volunteered to give back to their community. Several men participated in a 'buddy programme' through their local HIV community organisation with one participant describing his role as a 'friend counsellor'. Participants described how friends supported each other:

I still have a small cadre of friends that...we tend to interact when one of us gets sick. (Participant 2, age 67; LWH 24 years)

Participants also experienced challenges seeking out social support, describing difficulty making new friends, especially someone in their 'age bracket'. However, over time some developed new networks by joining organised activities such as a swimming group, seeking out walking 'buddies' or by exercising at the YMCA. Some were reluctant to ask for practical support as stated by this participant:

I've learned, this was a hard one for me, I've learned to ask for help. My friends have all said to me that, you know, don't be shy to say could you come over and give me a hand doing windows or something like that. (Participant 6, age 65; LWH 20 years)

\section{DISCUSSION}

Overall this study portrays a positive picture of older men living with HIV who integrate living strategies into their lifestyle to address their disability over time. Actively 
engaging in problem-solving by prioritising and modifying activities and avoiding stress, advocating for support, being positive and future oriented, engaging in healthy pursuits and providing social support to others helped men to mitigate their disability. Self-care and social support strategies were also found to be important in a cross-sectional Canadian study examining approaches to successful ageing in people living with HIV. ${ }^{30}$ By following participants over time, we gathered insights into triggers of episodes of disability and their perceptions of the success of implementing their living strategies and avoiding uncertainty.

While many studies of people living with HIV have identified the value of receiving social support, few have examined the benefits of providing social support. Our findings that men valued reciprocity of social support reinforces Emlet et al's notion of generativity, or a sense of well-being for future generations, as a component of resilience or the successful adaptation to negative life events. ${ }^{31}$ Participants in our study described reasons beyond "giving back" to the HIV community, including pragmatic reasons such as caring for one's parents and as a way of diverting attention from their own problems.

Rosenfeld et al found that even people living with HIV with strong social support from those who are HIV negative require the mutual understanding that comes from individuals living with HIV and HIV community organisations. ${ }^{32}$ The important role of HIV community organisations in facilitating links to social supports, and providing opportunities for adults ageing with HIV to reciprocate their support to others, was reinforced in this study. Interestingly, avoidance of HIV support groups was identified as a strategy to support well-being by shifting the focus away from HIV. ${ }^{32}$ This emphasises the need for HIV community organisations to understand clients' needs and goals and tailor their support services accordingly.

Although a well-established approach for many chronic illnesses, self-management programmes for people living with HIV are still emerging as a way to develop living strategies. This study supports the importance of programmes that promote self-management for people living with HIV in several ways. Participants in this study learnt to identify triggers of episodes of illness or disability to proactively mitigate severity of disability. This was important for setting realistic goals, a key component of self-management programmes, ${ }^{33}$ and for prioritising and modifying their tasks and activities. Living strategies that include problem-solving can help build self-efficacy, increase one's sense of control and mitigate feelings of uncertainty.

The ability to communicate effectively with health providers and advocate for increased access to health services is another common component of self-management programmes. ${ }^{33}$ The participants were knowledgeable about HIV and the health system and described how they were able to advocate for their needs over time. Health literacy is important to be able to navigate the health system and knowledgeably express one's needs. ${ }^{34}$ It is possible that many of the long-term survivors had to learn to advocate for supports at a time when knowledge of HIV was emerging and stigma was widespread. Regardless, increasing HIV knowledge and assisting people living with HIV with communicating their needs may be an important goal to enable better navigation of the health system for timely and appropriate access to resources.

The strategy of remaining future oriented with a positive outlook may be associated with resilience in those ageing with HIV. Resilience may be a modifiable factor which is protective against cognitive and functional decline in people living with HIV. ${ }^{35}$ Although further research is required to determine the causal relationship between resilience and function in people living with HIV, evidence suggests that interventions targeting resilience (eg, positive reframing and coping) may be an important component of managing disability in older adults with HIV. ${ }^{36}$ Self-efficacy is a protective factor which may moderate the negative consequences of living with HIV. ${ }^{37}$ Self-management strategies that increase self-efficacy for dealing with the episodic nature of HIV and associated uncertainty, and promote positive coping, may help develop resilience living with HIV.

Although present, uncertainty did not play as prominent a role in participants' disability experiences, in contrast to other studies. ${ }^{13}$ It is possible that the living strategies had been well-established and in use for some time by the men, and their attempts to pre-emptively identify triggers of disability helped them to avoid fluctuations in their health challenges and avoid uncertainty. People living with HIV also experience varied trajectories of episodic disability, one of which is described as stable ${ }^{38}$ Hence it is also possible that these participants experienced little fluctuation in their disability and less uncertainty over time.

Strengths of this study include the prolonged engagement and retention of all participants over the duration of this study which gave us insights into the living strategies used by people living with HIV over time. In addition, the use of a disability lens as a foundation for this research allows for understandings into the consequences of comorbidities and how environmental and personal factors influence living strategies over time.

This study is limited in that participants were recruited from HIV community service organisations and thus may be a more independent community-dwelling sample better able to access supports and actively engage in positive living strategies. As time since diagnosis has been shown to be positively associated with resilience and mastery, an affective resource that helps develop self-control, ${ }^{39}$ these long-term survivors may have been better able to cope with their episodic disability over time.

This research has several clinical implications. Self-management is recognised as an important element for developing long-term living strategies to 
manage chronic conditions, including HIV. ${ }^{33}$ Increased access to health services and improved communication with health providers are common outcomes of self-management programme. ${ }^{33}$ To achieve favourable health outcomes ageing with HIV, people living with HIV require training and support to effectively selfmanage their health, communicate their potential needs to access services and participate in shared decision-making with their healthcare providers.

Participants did not link two of the living strategies, valuing reciprocity of social support and looking to the future, directly to dealing with the episodic nature of disability. It may be that these strategies are dispositional in nature and represent an overall approach to dealing with health challenges or disability. Being future oriented with a positive outlook assists in dealing with adversity and promotes successful ageing in the general population, ${ }^{40}$ thus may minimise the impact of episodes of ill health and associated stressors for people ageing with HIV. Similarly, productive engagement in work and volunteering is also associated with successful ageing. ${ }^{41}$ These findings reinforce the importance of promoting these strategies in people living with HIV through providing outlets to reciprocate social support and promoting strategies that facilitate positive cognitive coping.

Contributors PS and KKO developed the research question and designed the study. PS, KKO, RM and MS participated in the data analysis. PS drafted the manuscript. KKO, RM and MS contributed to the critical revision and redrafting of the manuscript.

Funding This work was supported by the Canadian Institutes of Health Research (ClHR) under grant HHP 131556. KKO is supported by a CIHR New Investigator Award.

Competing interests None declared.

Patient consent for publication Not required.

Ethics approval Research Ethics Board approvals were received from McMaster University and the University of Toronto. All participants provided written informed consent.

Provenance and peer review Not commissioned; externally peer reviewed.

Data availability statement № data are available.

Open access This is an open access article distributed in accordance with the Creative Commons Attribution Non Commercial (CC BY-NC 4.0) license, which permits others to distribute, remix, adapt, build upon this work non-commercially, and license their derivative works on different terms, provided the original work is properly cited, appropriate credit is given, any changes made indicated, and the use is non-commercial. See: http://creativecommons.org/licenses/by-nc/4.0/.

\section{REFERENCES}

1. O'Brien KK, Bayoumi AM, Strike C, et al. Exploring disability from the perspective of adults living with HIV/AIDS: development of a conceptual framework. Health Qual Life Outcomes 2008;6:76.

2. Rusch M, Nixon S, Schilder A, et al. Impairments, activity limitations and participation restrictions: prevalence and association among persons living with HIV/AIDS in British Columbia. Health Qual Life Outcomes 2004;2:46.

3. Johs NA, Wu K, Tassiopoulos K, et al. Disability among middle-aged and older persons with human immunodeficiency virus infection. Clin Infect Dis 2017;65:83-91.

4. Thompson L, Abel G. The work of negotiating HIV as a chronic condition: a qualitative analysis. AIDS Care 2016;28:1571-6.
5. Public health agency of Canada. HIVIAIDS epi updates: national HIV prevalence and incidence estimates for 2011. Centre for Communicable Diseases and Infection Control, Public Health Agency of Canada, 2014.

6. Guaraldi G, Orlando G, Zona S, et al. Premature age-related comorbidities among HIV-infected persons compared with the general population. Clin Infect Dis 2011;53:1120-6.

7. Rueda S, Law S, Rourke SB. Psychosocial, mental health, and behavioral issues of aging with HIV. Curr Opin HIV AIDS 2014;9:325-31.

8. Emlet CA. An examination of the social networks and social isolation in older and younger adults living with HIV/AIDS. Health Soc Work 2006;31:299-308.

9. O'Brien KK, Davis AM, Strike C, et al. Putting episodic disability into context: a qualitative study exploring factors that influence disability experienced by adults living with HIV/AIDS. $J$ Int AIDS Soc 2009;12:30.

10. Rosenfeld D, Ridge D, Lob GV, et al. Vital scientific puzzle or lived uncertainty? Professional and lived approaches to the uncertainties of ageing with HIV. Health Sociology Review 2014;23:20-32.

11. Solomon P, O'Brien K, Wilkins S, et al. Aging with HIV: a model of disability. J Int Assoc Provid AIDS Care 2014;13:519-25.

12. O'Brien KK, Hanna S, Solomon P, et al. Characterizing the disability experience among adults living with HIV: a structural equation model using the HIV disability questionnaire (HDQ) within the HIV, health and rehabilitation survey. BMC Infect Dis 2019;19.

13. Solomon P, O'Brien K, Wilkins S, et al. Aging with HIV and disability: the role of uncertainty. AIDS Care 2014;26:240-5.

14. Furlotte C, Schwartz K. Mental health experiences of older adults living with HIV: uncertainty, stigma, and approaches to resilience. Can. J. Aging 2017;36:125-40.

15. Kylmä J, Vehviläinen-Julkunen K, Lahdevirta J. Hope, despair and hopelessness in living with HIV/AIDS: a grounded theory study. J Adv Nurs 2001;33:764-75.

16. Moskowitz JT, Hult JR, Bussolari C, et al. What works in coping with HIV? A meta-analysis with implications for coping with serious illness. Psychol Bull 2009;135:121-41.

17. Brashers DE, Neidig JL, Goldsmith DJ. Social support and the management of uncertainty for people living with HIV or AIDS. Health Commun 2004;16:305-31.

18. O'Brien KK, Dagenais $M$, Solomon $P$, et al. Use of living strategies among adults aging with HIV in Canada: comparison by age-group using data from the HIV, health and rehabilitation survey. J Int Assoc Provid AIDS Care 2018;17:2325958218774041.

19. Beard J, Feeley F, Rosen S. Economic and quality of life outcomes of antiretroviral therapy for HIV/AIDS in developing countries: a systematic literature review. AIDS Care 2009;21:1343-56.

20. Harocopos A, Dennis D. Maintaining contact with drug users over an 18-month period. Int J Soc Res Methodol 2003;6:261-5.

21. Solomon P, Wilkins S. Participation among women living with HIV: a rehabilitation perspective. AIDS Care 2008;20:292-6.

22. Gordillo V, Fekete EM, Platteau T, et al. Emotional support and gender in people living with HIV: effects on psychological well-being. J Behav Med 2009;32:523-31.

23. Vosvick M, Martin LA, Smith NG, et al. Gender differences in HIVrelated coping and depression. AIDS Behav 2010;14:390-400.

24. DeGrezia MG, Scrandis D. Successful coping in urban, communitydwelling older adults with HIV. J Assoc Nurses AIDS Care 2015;26:151-63.

25. Justice AC, Holmes W, Gifford AL, et al. Development and validation of a self-completed HIV symptom index. J Clin Epidemiol 2001;54:S77-S90.

26. QDA Miner 5.0. Provalis research, 2016.

27. Thomson R, Holland J. Hindsight, Foresight and insight: the challenges of longitudinal qualitative research. Int $J$ Soc Res Methodol 2003;6:233-44.

28. Strauss A, Corbin J. Basics of qualitative research. Newbury Park, CA: Sage, 1990.

29. Morse JM, Barrett M, Mayan M, et al. Verification strategies for establishing reliability and validity in qualitative research. International Journal of Qualitative Methods 2002;1:13-22.

30. Emlet CA, Harris L, Furlotte C, et al. 'I'm happy in my life now, I'm a positive person': approaches to successful ageing in older adults living with HIV in Ontario, Canada. Ageing Soc 2017;37:2128-51.

31. Emlet CA, Tozay S, Raveis VH. "I'm not going to die from the AIDS": resilience in aging with HIV disease. Gerontologist 2011;51:101-11.

32. Rosenfeld D, Catalan J, Ridge D, et al. Strategies for improving mental health and wellbeing used by older people living with HIV: a qualitative investigation. AIDS Care 2018;30:102-7. 
33. Bernardin KN, Toews DN, Restall GJ, et al. Self-Management interventions for people living with human immunodeficiency virus: a scoping review. Can J Occup Ther 2013;80:314-27.

34. Perazzo J, Reyes D, Webel A. A systematic review of health literacy interventions for people living with HIV. AIDS Behav 2017;21:812-21.

35. Fazeli PL, Moore RC, Vance DE. Resilience attenuates the association between neurocognitive functioning and everyday functioning in individuals aging with HIV in the deep South. Int $J$ Geriatr Psychiatry 2019;34:72-8.

36. Fumaz CR, Ayestaran A, Perez-Alvarez N, et al. Resilience, ageing, and quality of life in long-term diagnosed HIV-infected patients. AIDS Care 2015;27:1396-403.

37. Emlet CA, Fredriksen-Goldsen KI, Kim H-J. Risk and protective factors associated with health-related quality of life among older gay and bisexual men living with HIV disease. Gerontologist 2013;53:963-72.
38. Solomon P, O'Brien KK, Nixon S, et al. Trajectories of episodic disability in people aging with HIV: a longitudinal qualitative study. $J$ Int Assoc Provid AIDS Care 2018;17.

39. Emlet CA, Shiu C, Kim H-J, et al. Bouncing back: resilience and mastery among HIV-positive older gay and bisexual men. Gerontologist 2017;57(suppl 1):S40-S49.

40. Klugar M, Čáp J, Klugarová J, et al. The personal active aging strategies of older adults in Europe: a systematic review of qualitative evidence. JBI Database System Rev Implement Rep 2016;14:193-257.

41. Kail BL, Carr DC. Successful aging in the context of the disablement process: working and volunteering as moderators on the association between chronic conditions and subsequent functional limitations. $J$ Gerontol B Psychol Sci Soc Sci 2017;72:340-50. 